\title{
Relative importance of herd-level risk factors for probability of infection with paratuberculosis in Irish dairy herds
}

\author{
Conor G. McAloon, ${ }^{* 1}$ Michael L. Doherty, ${ }^{*}$ Paul Whyte, ${ }^{*}$ Simon J. More, ${ }^{*}$ Luke O'Grady, ${ }^{*}$ Lorna Citer,† \\ and Martin J. Greenł \\ *Section of Herd Health and Animal Husbandry, School of Veterinary Medicine, University College Dublin, Belfield, Dublin 4, Ireland, D04 W6F6 \\ †Animal Health Ireland, Carrick-on-Shannon, Co. Leitrim, Ireland, D04 W6F6 \\ $\ddagger$ School of Veterinary Medicine and Science, University of Nottingham, Sutton Bonington, United Kingdom, LE12 5RD
}

\section{ABSTRACT}

Control of paratuberculosis is challenging due to the relatively poor performance of diagnostic tests, a prolonged incubation period, and protracted environmental survival. Prioritization of herd-level interventions is not possible because putative risk factors are often not supported by risk factor studies. The objective for this study was to investigate the relative importance of risk factors for an increased probability of herd paratuberculosis infection. Risk assessment data, comprehensive animal purchase history, and diagnostic test data were available for 936 Irish dairy herds. Both logistic regression and a Bayesian $\beta$ regression on the outcome of a latent class analysis were conducted. Population attributable fractions and proportional reduction in variance explained were calculated for each variable in the logistic and Bayesian models, respectively. Routine use of the calving area for sick or lame cows was found to be a significant explanatory covariate in both models. Purchasing behavior for the previous $10 \mathrm{yr}$ was not found to be significant. For the logistic model, length of time calves spend in the calving pen (25\%) and routine use of the calving pen for sick or lame animals (14\%) had the highest attributable fractions. For the Bayesian model, the overall $\mathrm{R}^{2}$ was $16 \%$. Dry cow cleanliness $(7 \%)$ and routine use of the calving area for sick or lame cows $(6 \%)$ and had the highest proportional reduction in variance explained. These findings provide support for several management practices commonly recommended as part of paratuberculosis control programs; however, a large proportion of the observed variation in probability of infection remained unexplained, suggesting other important risks factors may exist.

Key words: paratuberculosis, Johne's disease, risk factor

Received April 5, 2017.

Accepted July 25, 2017.

${ }^{1}$ Corresponding author: mcaloonconor@gmail.com

\section{INTRODUCTION}

Bovine paratuberculosis, also called Johne's disease (JD), is characterized by chronic granulomatous enteritis, which manifests clinically as a protein-losing enteropathy causing diarrhea, hypoproteinaemia, emaciation, and eventually death (Sweeney et al., 2012). Adverse effects on animal productivity in terms of lower milk yield (McAloon et al., 2016), higher cull rates (Hendrick et al., 2005), reduced value for culled animals (Richardson and More, 2009), possible adverse effects on fertility (Johnson-Ifearulundu et al., 2000), and losses due to continued spread of infection are key drivers in the attempt to control the disease at the farm level. In addition, some research exists to suggest that the etiologic pathogen Mycobacterium avium ssp. paratuberculosis (MAP) may pose a zoonotic risk (Chiodini et al., 2012).

Control of JD is difficult due to the relatively poor performance of diagnostic tests (Nielsen et al., 2008), a prolonged incubation period (Sweeney et al., 2012), and protracted environmental survival (Whittington et al., 2004). Several simulation studies have concluded that test and cull programs are unlikely to be effective in isolation and that control of the disease on farm should center primarily on closing infection routes, ideally in combination with testing and culling ( $\mathrm{Lu}$ et al., 2010; Kudahl et al., 2011; Robins et al., 2015). However, there is little empirical evidence to support many of the specific interventions introduced at the herd level to reduce the probability of introduction and transmission of disease. Although several risk factor studies have been conducted, results often fail to agree with putative risk factors that inform key aspects of control programs, making prioritization of implementable control measures difficult (McAloon et al., 2015).

At least part of the disparity in these studies may be due to misclassification of positive and negative herds. Conventionally, herd level risk factor studies are conducted by attributing an infection status to each herd based on a set number of test reactors. However, 
such dichotomized approaches may discard important information regarding the likelihood of infection and may be biased in larger herds due to imperfect test specificity ( $\mathbf{S p})$.

The use of Bayesian latent class methods allows the estimation of a probability of infection for each herd conditional on the test characteristics, number of test positive animals, and total number of animals in the herd (Branscum et al., 2004). In addition, Bayesian methods account for uncertainty associated with model parameters by modeling each parameter as a random variable with an associated probability distribution. Bayesian inference allows direct inference on the parameter of interest, conditional on the observed data and the prior distributions (Messam et al., 2008).

In Ireland, control of nonstatutory diseases such as JD is coordinated by Animal Health Ireland (AHI; More et al., 2011). In 2013, a pilot voluntary Johne's Disease Control Programme was introduced, which combined annual testing of all animals over 24 mo of age with an on-farm risk assessment and management plan (RAMP) that captured herd management practices relevant to JD. The RAMP has been widely adopted across many countries with recognizable control programs (Geraghty et al., 2014). The risk assessment (RA) component involves assigning risk scores to different management procedures and areas based on observations and farmer reported practice. In addition, within the Irish system, animal movement data for the herd are provided for the practitioner to assess bioexclusion risks. The outcome of the RA is used to inform a management plan and, in national programs, may have some bearing on herd categorization or herd risk score.

A reduction in animal-level test positivity associated with the implementation of management practices has been found in several small-scale investigations on demonstration or study herds using the RAMP approach (Ferrouillet et al., 2009; Pillars et al., 2011; Espejo et al., 2012), but progress has not been reproduced in larger studies on commercial farms (Sorge et al., 2011). Furthermore, to the authors' knowledge no studies are available investigating the risk associated with RAMP scores in combination with comprehensive herd purchase history, or modeling herd level infection status on a probabilistic scale.

The objective of this study was to identify and evaluate the relative importance of risk factors for JD probability of infection using diagnostic test results, RA scores, and animal movement history for herds enrolled in the national voluntary Johne's Disease Control Programme.

\section{MATERIALS AND METHODS}

\section{Data Set}

The data set for the current study was obtained from herds enrolled in the national voluntary Johne's Disease Control Programme. Enrolled herds were required to have all animals that were 24 mo of age and older serologically tested using either serum or milk samples.

Diagnostic testing was conducted in approved government and commercial laboratories using 1 of 3 commercial ELISA kits approved for use in the AHI program: Parachek (Prionics, Switzerland), Paratuberculosis Antibody Screening Test (Idexx, Westbrook, ME), and ID Screen (IDVet, Montpellier, France). Producers that elect to test using blood or milk sample were required to test all eligible animals once or twice per year, respectively. Test data were stored centrally in the Irish Cattle Breeding Federation computer database. Data were extracted for the period beginning November 1, 2013, and ending December 30, 2014, and included anonymized cow and herd identifiers, test date, sample-topositive $(\mathbf{S} / \mathbf{P})$ ratio, laboratory interpretation (negative, suspect, positive), sample type (blood or milk), testing laboratory (test kit), and county. Diagnostic test data were available for 1,040 herds.

Given that the time frame for extraction exceeded 12 mo, several herds had results from more than one herd screen. To reduce the potential for reverse causality (i.e., the effect of changes in management occurring as a result of a positive diagnosis), the last herd screen occurring before the RAMP was preferentially selected, followed by the soonest herd screen occurring after the RAMP. Test and animal movement data were extracted separately and data sets were aligned using coded herd identifiers. An additional binary variable was created to investigate the effect of having the test before (1) versus after (0) the RAMP. The values for sensitivity (Se) and Sp used in the models were appropriate for a single test.

In addition to ongoing testing, enrolled herds were required to have an annual RAMP carried out by a program-approved veterinary practitioner. The RAMP contained questions on the history of the disease on each farm as well as the risk of introduction of infection from sources other than animal movement (e.g., colostrum, slurry contractors, and so on). The biocontainment component of the RAMP consisted of an additional 28 questions regarding management practices and observations made on the farm at the day of the visit, which were deemed to be relevant to the spread of JD. 
In the RA used in the Irish program, questions were scored using an ordinal scale of $1,4,7$, and 10 . Within the AHI program, this method was used to reduce the potential for subjectivity that might be associated with the use of a continuous scale, because each specific management practice may be associated with a particular score on the ordinal scale. In addition, the use of $1,4,7$, and 10 rather than $1,2,3$, and 4 was used as a means of weighting the risk associated with each management practice. Higher scores were associated with increased risk of transmission. However, for this study, RA scores were modeled as unordered categorical variables, thereby reflecting the risk associated with specific practices rather than the risk scores per se and ensuring that the original scale used would have no effect on the model outcome. Questions asked as part of the RAMP are shown in Table 1.

To assist in assessing bioexclusion, the RAMP was pre-populated with animal movement data for the herd over the preceding $10 \mathrm{yr}$. Movement data included herd size, number of male and female introductions, number of source herds, and number of overseas imports for every year from 2005 to 2014 . Herd sizes less than 20 in 2014 were dropped from the analysis. Herd size was next summarized across the 10-yr period: herds that had a herd size of $<105 \%$ of herd size in 2005 were categorized as nongrowing herds; the remaining herds were then broken into mild, moderate, and large growth by categorizing the percentage growth into 3 equal quantiles: 5 to $25 \%, 26$ to $46 \%$, and $>46 \%$.

Movement and herd size data were then aggregated over two 5-yr periods, 2005 to 2009 and 2010 to 2014 . Within each 5-yr period, herds were described as "closed" if no purchases had been made, herds where no females were purchased and males were purchased at $<5 \%$ of the overall herd size were described as "replacement bulls only," for the remaining herds, the number of female purchases was averaged across the 5-yr pe-

Table 1. Questions asked as part of an on-farm risk assessment (RA) conducted on 925 dairy herds enrolled in the Irish national Johne's Disease Control Programme

1. Have you ever completed a Johne's disease (JD) herd test?

2. Has there been any suspect cases of JD on the farm?

3. Have you had any confirmed clinical JD or test positive cows in your herd?

4. Do you use your own equipment to spread slurry on your farm?

5. Do you spread cattle/slurry from other herds on your pasture?

6. Do you graze cattle purchased by you for fattening on your pasture?

7. Do you graze cattle/cows on commonage or with cattle from other herds?

8. Do you graze on rented ground?

9. Do you use contract rearers or rear calves/heifers under a different herd number?

10. Do sheep cograze on this farm?

11. Are calves fed colostrum from own mother or from known low risk colostrum cows or artificial?

12. Are at least $3 \mathrm{~L}$ of colostrum (first milking) consumed within the first $2 \mathrm{~h}$ ?

13. Are calves fed on low risk whole milk?

14. How often is non-saleable whole milk (high risk) fed?

15. Are calves housed in individual or group pens in the first week?

16. Is there exposure to cow manure in the calf housing or grazing area?

17. Is there exposure to cow manure by watering or feeding utensils?

18. Are calves fed forages that have received slurry from adult animals within the last year?

19. Do you feed or have you fed colostrum from other herds?

20. When was this last fed?

21. Do you feed milk from cows from other herds?

22. When was this last fed?

23. Are weaned heifers exposed to cows or their manure at any time?

24. Are maiden heifers exposed to cows or their manure at any time?

25 . What is the overall hygiene and cleanliness score of weaned heifers?

26. What is the overall hygiene and cleanliness score of maiden or incalf heifers?

27. Are weaned heifers $(>6$ months) fed forages that have received slurry from adult animals within the last year?

28. Are maiden or incalf heifers ( $\geq 6$ months) fed forages that have received slurry from adult animals within the last year?

29. Dry cow area environment hygiene score

30. Milking cow area environment hygiene score

31. Dry cow cleanliness

32. Milking cow cleanliness

33. Single or multiple cows in calving areas?

34. Manure buildup

35. Manure on soiled udders and legs of cows?

36. Calving area used for lame or sick cows?

37. Calving area used for JD clinical or JD test positive cows?

38. Birth of calves in areas other than designated calving area?

39. Likelihood of calf nursing cow(s)?

40. How fast are newborn dairy calves removed from their mothers? 
riod and broken into 3 equal quantiles: low, medium, and high replacement purchase. Given small number of herds were in the "closed" category for each 5-yr period, this category was combined with "replacement bulls only" for the analysis. An additional binary variable was created to identify herds where males were purchased at greater than $5 \%$ of the overall herd size. These herds were considered likely to be purchasing male animals for beef production in addition to the dairy enterprise. Finally, the number of source herds purchased from each year was averaged across each 5-yr period.

Herds were removed from the data set when one or more of the 3 components of the scheme (diagnostic test results, herd movement history, and RAMP results) were missing or incomplete. The final data set included data from 925 herds.

\section{Analytical Models}

Model 1; Logistic Regression Analysis. The outcome variable was herd infection status (positive or negative) and herds were defined as positive when they had 2 or more cows with positive tests. A cut point of 2 positive cows was used to account for imperfect test Sp; with herd sizes represented in this study, it was less likely that 2 positive results would both be false positives. In addition, for the purposes of comparison, the final model was reassessed with a cut point of 1 reactor, the final single reactor model is included as supplemental material (Supplemental Table S1; https://doi.org/ 10.3168/jds.2017-12985). Data analysis was conducted in R-studio version 1.0.44 (R Core Team, 2016). Individual explanatory covariates were initially investigated within a univariable logistic regression framework and carried forward for multivariable regression analysis when $P<0.2$. Before addition to the multivariable model, covariates were assessed for correlation. When 2 variables were highly correlated $(>0.8)$, one was selected and brought forward for multivariable analysis based on whichever variable resulted in the model with the lowest Akaike information criterion. Variables dropped due to collinearity were replaced into the final model to test for significance. The model was constructed using a forward stepwise elimination and variables with a significance probability $P<0.05$ were retained in the model. Herd size and test medium were forced into the model from the beginning of the multivariate analysis to account for the potential confounding effect of these variables on test Se and Sp. In addition, for the purpose of comparison, the model was reconstructed using the Akaike information criterion solely as the selection criterion. Finally, the population attributable fraction
(PAF) was calculated for each variable in the model based on distribution of exposure in cases (Hanley, 2001):

$$
\mathrm{PAF}=\frac{\mathrm{RR}-1}{\mathrm{RR}} \times \frac{\text { number of exposed cases }}{\text { overall number of cases }} .
$$

Adjusted relative risks (RR) were calculated from the odds ratios of the final model using the method described by Zhang and Kai (1998).

Model 2; Bayesian Analysis. This analysis was conducted in 2 stages. First, a probability of infection for each herd was estimated using a Bayesian latent class model. This model had the same structure and was implemented using the same methods as described in McAloon et al. (2016a). Briefly, the number of test positive animals in a given herd was assumed to follow a binomial distribution with a probability equal to the apparent prevalence (AP) and $\mathrm{n}$ equal to the number of animals tested. The AP was related to the true prevalence (TP) and the test Se and Sp by the formula

$$
\mathrm{AP}=\mathrm{TP} \times \mathrm{Se}+(1-\mathrm{TP}) \times(1-\mathrm{Sp}),
$$

where TP was modeled as a mixture of a Bernoulli distribution, with a probability equal to the probability of infection for the herd, and a $\beta$ distribution equal to the within-herd TP.

In the second step, the mean probability of infection for each herd was used as the outcome variable in a Bayesian $\beta$ regression model with a logit link (Branscum et al., 2007). The model was built using a forward stepwise analysis and variables were retained in the model when the $95 \%$ credible interval did not include zero.

The model had the following structure:

$$
\begin{gathered}
\mu_{\mathrm{i}} \sim \beta\left(\mathrm{a}_{\mathrm{i}}, \mathrm{b}_{\mathrm{i}}\right), \\
\mathrm{a}_{\mathrm{i}}=\psi_{\mathrm{i}} \times \gamma, \\
\mathrm{b}_{\mathrm{i}}=\left(1-\psi_{\mathrm{i}}\right) \times \gamma, \\
\operatorname{logit}(\psi)<-\beta_{0}+\beta_{1} \mathrm{X}_{1} \ldots, \\
\gamma \sim \operatorname{gamma}\left(\mathrm{G}_{1}, \mathrm{G}_{2}\right),
\end{gathered}
$$

where $\mu_{\mathrm{i}}$ was the probability of infection for the ith herd, which was modeled by a $\beta$ distribution with alpha and beta parameters $a_{i}$ and $b_{i}$, respectively. To facilitate incorporation of the covariate information into the regression model, the $\beta$ distribution was parameterized 
in terms of its mean, $\psi_{i}$, and a parameter related to its variance, $\gamma$ (Branscum et al., 2007). A logit link was used to estimate the regression coefficient, $\beta$, for each covariate, $\mathrm{X}$. The $\mathrm{G}_{1}$ and $\mathrm{G}_{2}$ were the shape and scale parameters for the gamma distribution, $\gamma$. Larger values of $\gamma$ correspond to less heterogeneity in the data. For this analysis, a prior gamma distribution with a low mean and high variance was used $\left(\mathrm{G}_{1}=\mathrm{G}_{2}=0.01\right)$.

Diffuse normal distributions (mean $=0$, precision $=0.01$ ) were used for the priors of each coefficient in the model. Model outcomes for each covariate were reported as probability of infection by converting the coefficients according to the formula

$$
p=\frac{1}{1+e^{-\left(\beta_{0}+\Sigma \beta_{\mathrm{j}} \mathrm{X}_{\mathrm{j}}\right)}},
$$

where $p$ is the probability of infection, $\beta_{0}$ is the intercept, and $\beta_{\mathrm{j}}$ is the coefficient of the $\mathrm{jth}$ covariate, $\mathrm{X}_{\mathrm{j}}$ (Dohoo et al., 2010). To fit the model, mean probabilities of infection less than $0.01(\mathrm{n}=8)$ were rounded to 0.01 and those greater than $0.99(\mathrm{n}=100)$ to 0.99 .

Model fit was assessed using posterior predictive simulations (Gelman et al., 2000). The predictive simulation incorporated within the model was

$$
\operatorname{Pred} \mu_{\mathrm{i}} \sim \beta\left(\mathrm{a}_{\mathrm{i}}, \mathrm{b}_{\mathrm{i}}\right) .
$$

Pred $\mu_{i}$ was monitored for the final 5,000 iterations of the overall simulation. Predicted probability of infection was compared with the probability of infection outcome from the latent class model and the mean difference, and the mean squared difference was used to compare models. The proportional reduction in variance explained was calculated for each variable by removing each variable in turn from the full model, re-estimating model parameters, and calculating the difference in $\mathrm{R}^{2}$ relative to the full model.

The model was implemented in WinBUGS 1.4.1 (Lunn et al., 2000), the first 5,000 iterations were discarded as burn-in, by which time convergence had occurred, and 15,000 iterations used for posterior inference. Convergence was assessed by visual assessment of the chain as well as by running multiple chains from dispersed starting values (Christensen et al., 2012). The code for analysis is provided as Supplemental Material (https://doi.org/10.3168/jds.2017-12985).

\section{RESULTS}

\section{Descriptive Statistics}

Nine hundred twenty-five herds were present in the final data set. Overall median herd size was 80 . Using a cut point of 2 reactors, 265 herds were positive, giving an AP of 0.29 . The RAMP scores are summarized in Figure 1, and animal purchase data for the $10 \mathrm{yr}$ before diagnostic testing are summarized in Table 2.

Median herd growth from 2005 to 2015 was 25\%. From 2005 to 2009, only 30 herds were classified as closed, with a further 70 herds classified as replacement bull purchases only. Similarly, from 2010 to 2014, 37 and 109 herds were closed or replacement bull only. From 2005 to 2009, 30\% of herds purchased replacement females at an annual average of more than 7.5\% of the total herd size, whereas from 2010 to 2014, the equivalent figure was $28 \%$. In each 5 -yr block, the mean

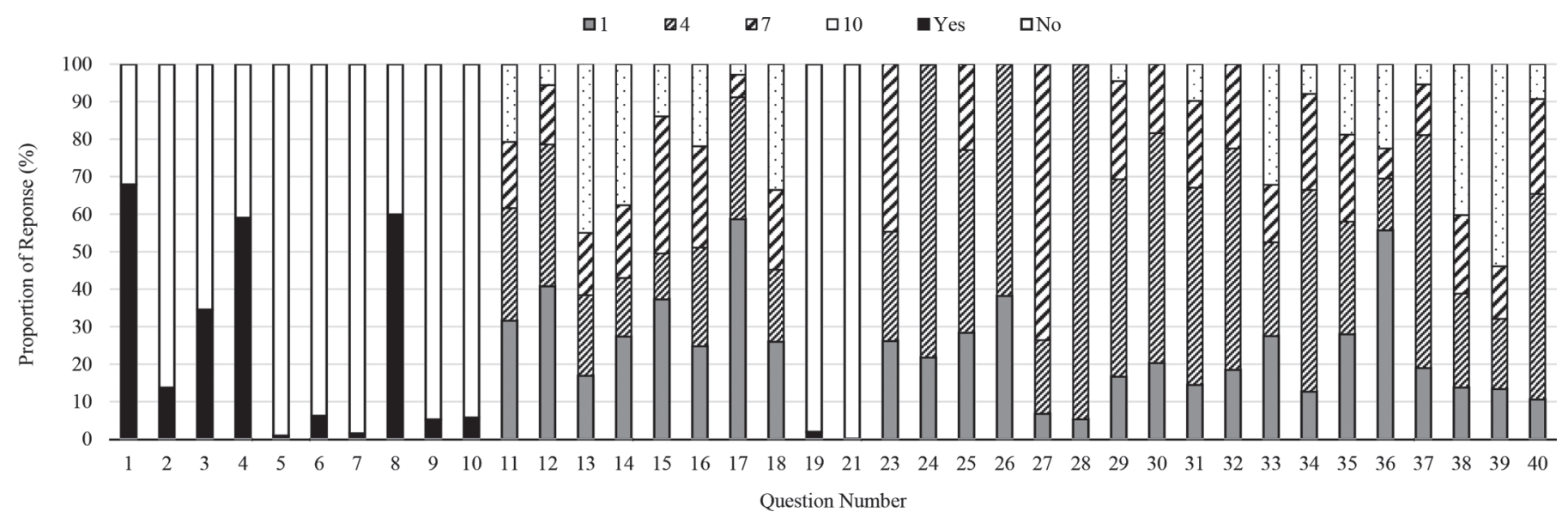

Figure 1. Stacked bar graph showing distribution of responses to risk assessment for 925 dairy herds enrolled in the Irish national Johne's Disease Control Programme. Questions 23 to 25 and question 17 are scored to a maximum 7, and questions 26 and 28 are scored to a maximum of 4 . 
annual number of source herds was more than 1 for $54 \%$ of the herds in 2005 to 2009 and $38 \%$ in 2010 to 2014 .

\section{Model Outcomes}

Model 1; Logistic Regression. The outputs from the final multivariable logistic regression model are shown in Table 3 . The reference category for each variable was selected to avoid negative coefficients. Herd size was positively associated with herd positivity with an odds ratio of 1.01 for each additional animal. Herds testing with milk were 1.57 times as likely to test positive as those testing with blood. A large seasonal effect was apparent, with herds testing in January 2.1 times as likely to test positive as those tested in May. Herds where pooled colostrum was used for more than $10 \%$ of the calves were 2.1 times as likely to be positive compared with those herds where calves were fed colostrum from their own test-negative dam; this category had a PAF of $11.6 \%$. Herds where weaned heifers were grazed near adult animals, but without direct or indirect contact, were 1.7 times as likely to test positive and had a PAF of $10.5 \%$, compared with those where direct or indirect contact was possible.

Table 2. Summary of herd-level characteristics and animal introduction data for 925 dairy herds enrolled in the Irish national Johne's Disease Control Programme ${ }^{1}$

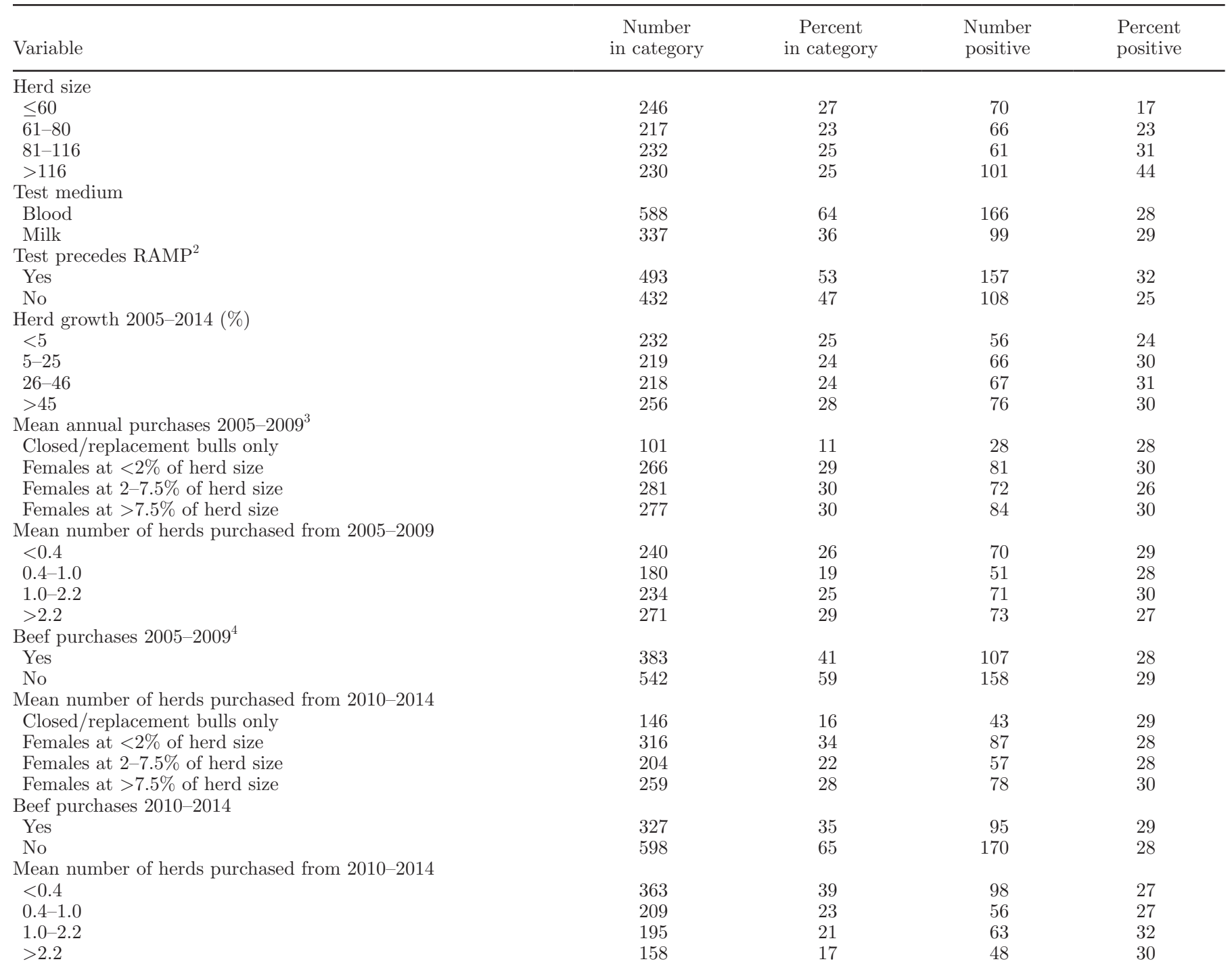

${ }^{1}$ Definition of categories and proportion of herds defined as positive were based on $\geq 2$ animals testing positive.

${ }^{2} \mathrm{RAMP}=$ risk assessment and management plan.

${ }^{3}$ Replacement bulls only $=$ herds not introducing females and only introducing males at $\leq 5 \%$ of the overall herd size each year.

${ }^{4}$ Beef purchases $=$ herds purchasing males at $>5 \%$ of the overall herd size each year. 
Herds where the milking cow environment had clearly visible manure contamination were 1.7 times as likely to be defined as positive compared with herds where only trace amounts of manure were visible with a PAF of $7.6 \%$. Herds where the calving area was routinely used for housing sick and lame cows were 2.2 times as likely to be positive than those where the calving area was never used for noncalving cows and had a PAF of $14.2 \%$. Herds where more than $50 \%$ of the calves were removed from the dam within $30 \mathrm{~min}$ of birth were 2.3 times as likely to test positive as those where $90 \%$ of the calves were removed within 15 min of birth; the PAF of this variable was $24.7 \%$. Finally, herds that experienced small growth (5-25\%) were 1.7 times as likely to test positive as those that expanded to a high $(>50 \%)$ growth in herd size.

Model 2; Bayesian Model. Outputs from the final Bayesian $\beta$-regression model are shown in Table 4. Overall, the model explained $16 \%$ of the variation, indicating that a considerable amount of the variation in the probability of a herd being positive remained unexplained (Figure 2). The reported presence of previous clinical or test positive animals was responsible for $22.6 \%$ of the overall variance explained $\left(\mathrm{R}^{2}\right)$ and resulted in a mean probability of infection (95\% probability interval) of $0.72(0.66-0.77)$. A strong seasonal effect was again observed, which was responsible for $35 \%$ of the overall $\mathrm{R}^{2}$, with herds testing in January having a probability of infection of $0.77(0.69,0.83)$. The proportion of the herd tested comprised $3.2 \%$ of the overall $\mathrm{R}^{2}$ and was negatively associated with the probability of infection. The probability of infection dropped by $5 \%$ with each additional $10 \%$ of the herd tested. Feeding of forages to weaned heifers that had been spread with slurry in the previous season increased the probability of infection by $8 \%(0-16 \%)$. Dry cow cleanliness comprised $7.1 \%$ of the overall $\mathrm{R}^{2}$ and herds where dry cows had no fecal contamination visible had a mean probability of infection of 0.67 , compared with 0.60 in herds where fecal contamination was visible on the legs but not extending above the dewclaws. The use of the calving pen for noncalving animals comprised $5.8 \%$ of the overall $\mathrm{R}^{2}$, and herds where the calving pen was routinely used for sick or lame animals had a probability of infection of $0.69(0.65-0.74)$.

\section{DISCUSSION}

The present study used a combination of frequentist and Bayesian methods to investigate risk factors for positivity and infection probability in Irish dairy herds using data collected as part of the AHI voluntary program.
In the logistic regression model, the speed with which calves were removed from the calving pen was the most important variable $(\mathrm{PAF}=24.7 \%)$. Herds in which $>90 \%$ calves were removed within 15 min of birth had the lowest risk of being positive, with herds where calves were still removed within 30 min were 2.2 times as likely to be positive. In this case, the large PAF is caused by a combination of the relatively large odds ratio, combined with the large proportion of herds within the higher risk category $(n=507)$. The practice of removing the calf immediately from the dam is commonly advocated for the purpose of paratuberculosis control; however, despite investigating this risk factor, several studies have failed to find this practice associated with an increased risk of positivity (Johnson-Ifearulundu and Kaneene, 1998; Wells and Wagner, 2000; Nielsen and Toft, 2011). However, Cashman et al. (2008), found an increased risk of culturing MAP from milk filters in herds where a greater proportion of calvings were not supervised. Interestingly, the practice of immediate separation from the dam is also recommended for the control of calf diseases (McGuirk and Collins, 2004), although studies into the benefit of calf removal have been equivocal (Weary and Chua, 2000; Trotz-Williams et al., 2007), McAloon et al. (2016b) recently found improved passive transfer in calves removed immediately from the calving pen, compared with those spending more than 30 min with the dam.

The use of the calving pen to house sick or lame animals was the second most important management factor in both the Bayesian and logistic model with a proportional reduction in $\mathrm{R}^{2}$ of $5.8 \%$ and a PAF of $14.2 \%)$. Herds that routinely used the calving pen for sick or lame cows had a mean probability of infection of $0.69(0.65-0.74)$ and were 2.3 times as likely to be defined as positive compared with herds in which the calving pen was never used for sick or lame cows. The use of the calving pen for sick or lame cows is often discouraged as part of JD control programs (Sweeney et al., 2012). This is based on the rationale that cows that are subclinically infected with JD are more likely to be susceptible and therefore affected with other diseases but there appears to be little empirical evidence to support this claim. It is, however, likely that sick cows would also include those suffering from symptoms of clinical JD and this practice could facilitate disease transmission. In addition, routine use of the calving pen for sick animals could be an indicator of increased stocking density and insufficient building space, potentially resulting in increased exposure of calves to infected fecal material.

The source of colostrum was significant in the logistic regression model and had a PAF 11.6\%. However, 
this variable was not significant in the Bayesian model. Herds in which over $10 \%$ of calves were fed colostrum from sources other than the dam (risk score 10) were

2.1 times as likely to be defined positive compared with herds in which dam-only colostrum was practiced. Nielsen et al. (2008) found that calves fed colostrum

Table 3. Results from multivariable logistic regression model assessing the association between risk assessment (RA) questions (Q), animal movement data, and the outcome herd positivity, defined as herds with 2 or more positive animals in the herd

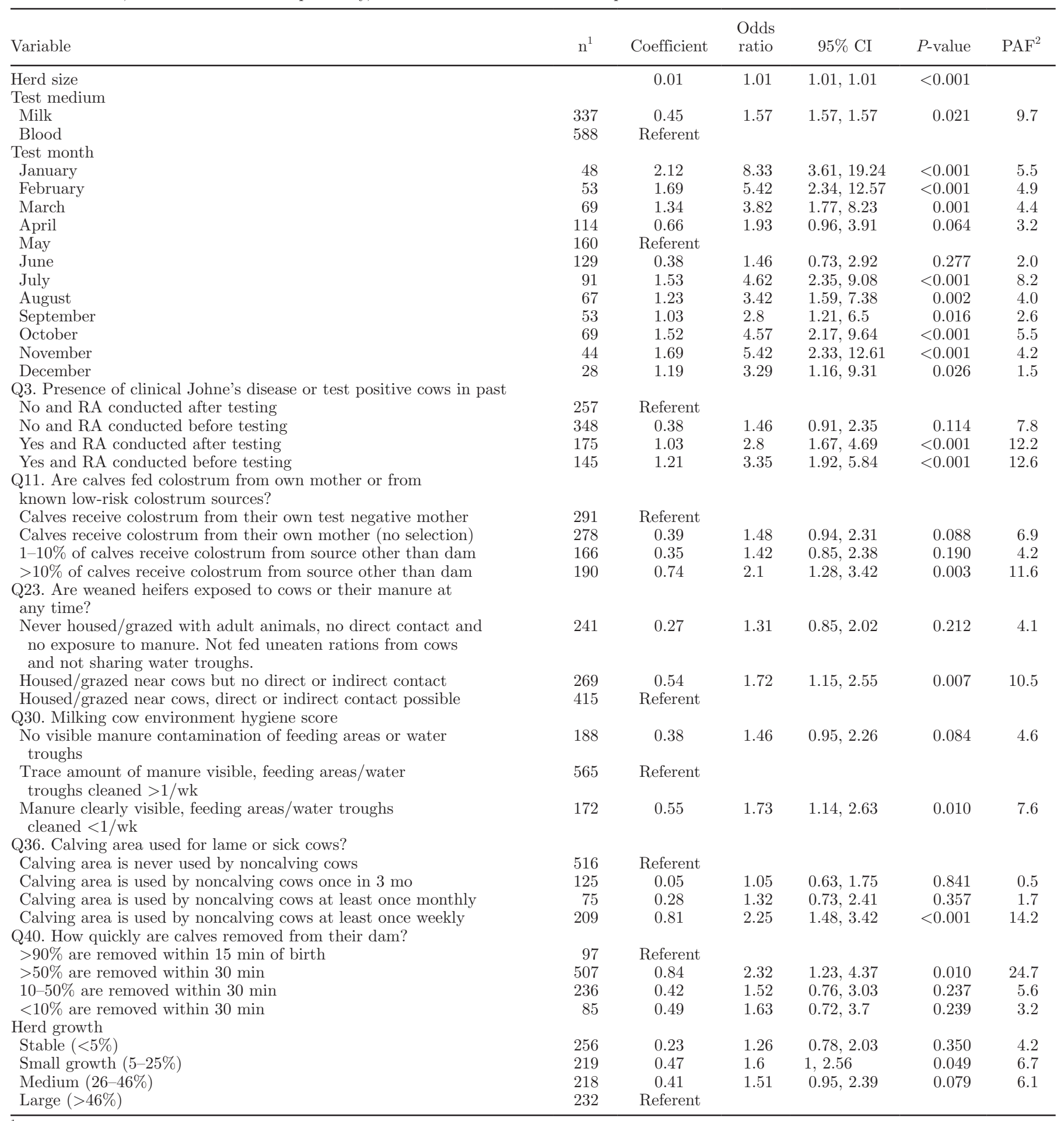

${ }^{1} \mathrm{n}=$ number in category.

${ }^{2} \mathrm{PAF}=$ population attributable fraction. 
from multiple sources were 1.2 times as likely to be positive than those fed dam-only colostrum. However, this finding is not consistent. For example, in a longitudinal study, Pithua et al. (2011) found that calves fed PCRpositive colostrum were not at a significantly greater risk of testing positive as adults compared with those fed PCR-negative colostrum. In contrast, the same author found that calves fed a commercial colostrum replacer were less likely to be identified as positive as adults than those fed conventional colostrum (Pithua et al., 2009). Similarly, Stabel (2008) found that colostrum pasteurization reduced the incidence of disease in calves as measured by interferon gamma. However, in the long term, risk of infection for this cohort of calves was not different between groups (Godden et al., 2015).

Dry cow cleanliness was significantly associated with probability of infection in the Bayesian model and was responsible for a reduction in $\mathrm{R}^{2}$ of $7.1 \%$ ). The finding that the lowest dry cow contamination score was associated with an increased risk of infection compared with the second lowest score seems counterintuitive. This finding could potentially be explained given the seasonal calving system operated on Irish dairy herds (i.e., the fact that the dry cow pen is not in use for a large majority of the year). However, whenever the month when the RAMP was conducted was forced into

Table 4. Results from final multivariable Bayesian $\beta$ regression model assessing the association between risk assessment (RA) questions (Q), animal movement data, and the probability of infection as estimated by a Bayesian latent class analysis

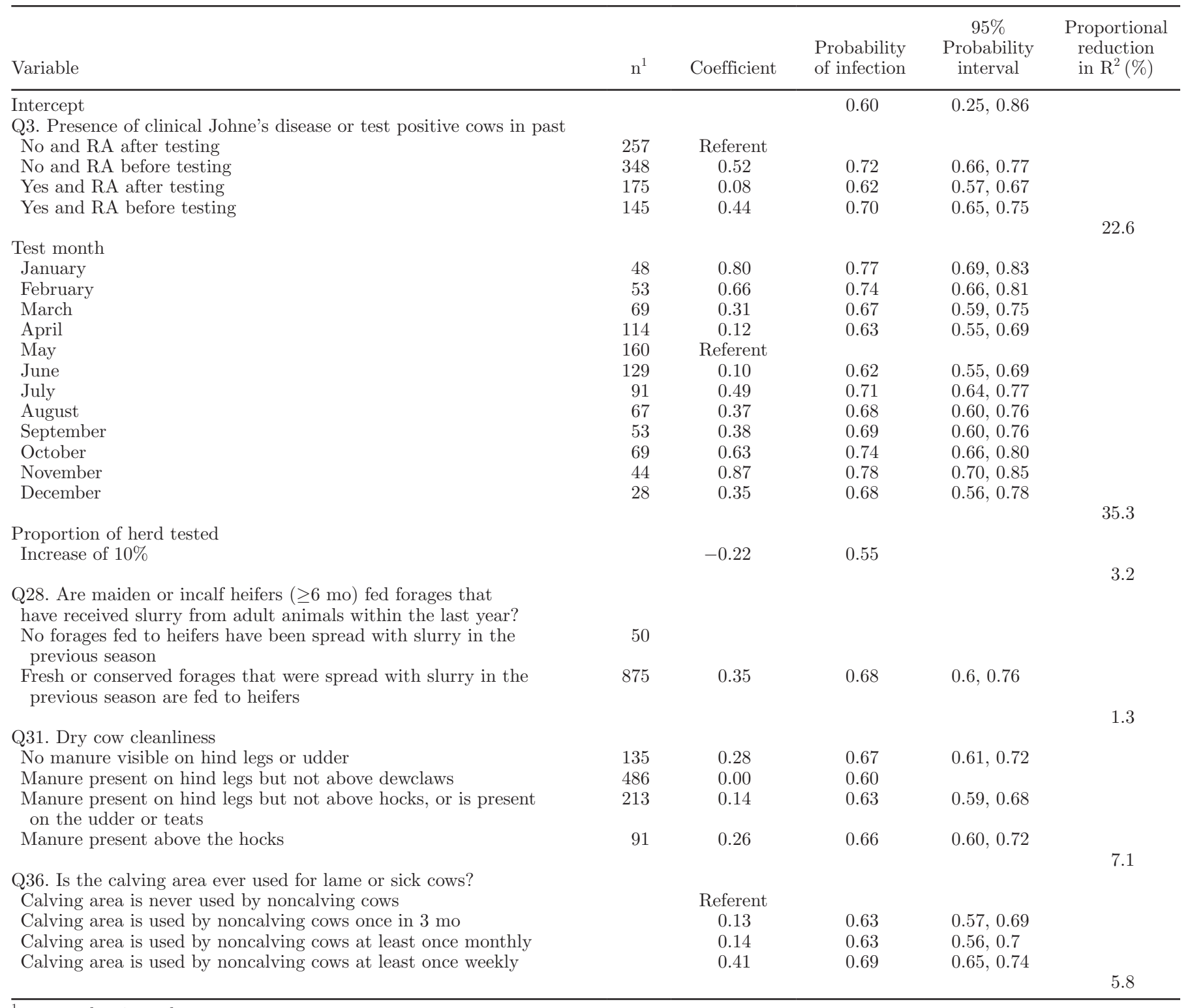

\footnotetext{
${ }^{1} \mathrm{n}=$ number in each category.
} 
the model, the variable remained significant, suggesting that the time when the RAMP was conducted was not confounding this variable. It is worth noting that risk scores of 7 and 10 were associated with increased risk compared with risk score 4 although these associations were not significant.

Similarly, the finding that herds where heifers were housed or grazed near cows but had no direct contact (question 23) were at greater risk of testing positive compared with those where there was direct contact or heifers were exposed via run-off or slurry spreading is difficult to explain. The susceptibility to infection has been shown to decrease with age (Windsor and Whittington, 2010); however, more recently, Mortier et al. (2013) demonstrated that calves up to the age of 12 mo could be infected with both high and low doses of MAP. Despite been identified as the lowest risk category for this model, the large proportion of herds where weaned heifers had direct or indirect contact with adult cows $(45 \%)$ is a significant concern.

The milking cow environment score had a PAF of $7.6 \%$ with herds where manure was clearly visible were 1.7 times as likely to test positive as those where trace amounts of manure was visible. Although infection of adult animals is possible with sufficiently high doses of MAP (Whittington and Windsor, 2010), in this case it is more likely that the finding is indicative of the overall hygiene of the farm, rather than the specific risk to adult animals per se.

In the Bayesian model, the feeding of forages that had received slurry from adult animals was significantly associated with the probability of infection; however, this variable only comprised $1.3 \%$ of the overall variation. Interestingly, a similar finding was observed in a North American study (Obasanjo et al., 1997). In

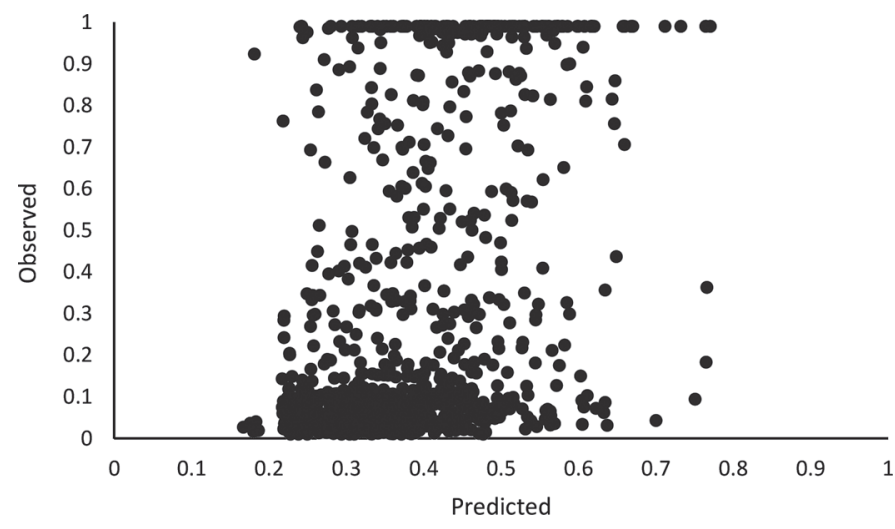

Figure 2. Predicted median probabilities of infection from the final Bayesian $\beta$ regression model plotted against estimated (observed) probabilities of infection from the Bayesian latent class model for each of the 925 herds enrolled in the Irish national Johne's Disease Control Programme. $\mathrm{R}^{2}=0.16$. contrast, Kohl et al. (2010) was unable to culture MAP from baled grass silage following inoculation, although samples were positive by PCR. The authors in that study suggested that conserved forages constituted a minor risk for transmission. In a pasture-based system where conserved forages are consumed during the housed period, avoiding the use of slurry on harvested grass may difficult to avoid, which is reflected in the high proportion of herds in the higher risk category (95\%). In addition, on many farms, avoiding spreading slurry on grass harvested for younger animals would necessitate segregation of conserved forage for different age groups of animals. Furthermore, increased application on adult ground would lead to an increase in potassium content (Soder and Stout, 2003), resulting in an increased DCAD and therefore an increased risk of hypocalcemia (Goff, 2004).

The change in herd size from 2005 to 2014 was only significant in the logistic model with a PAF of $6.7 \%$. In that case, the lowest risk of testing positive was observed in herds that had undergone significant expansion $(>50 \%)$ over the 10-yr period. Anecdotally, herd expansion has been associated with an increased risk of poor heath in general. However, in a previous Irish study, Jago and Berry (2011) found improved reproductive performance in dairy herds undergoing higher levels of expansion, suggesting that this finding could be confounded by improved management in general on these farms. In addition, the same study found that the average parity number decreased in herds as the rate of expansion increased. The Se of the ELISA is known to increase with increased age (Nielsen et al., 2013); therefore, as the mean age of the herd decreases, the effective herd-level Se of the ELISA screen is also likely to have decreased.

In the Bayesian model, previous presence of test positive or clinical cases of JD explained the largest proportion of variance explained (41\%); however, in the logistic model, this variable had a PAF of $12.6 \%$. The finding is unsurprising and highlights awareness of the herd infection status in many herds. It was decided to couple this variable with whether or not the RAMP had been conducted before or after the herd screen in an attempt to remove any possible confounding associated with prior knowledge of the disease in the herd. When the variable was removed from the model, all of the variables remained significant.

Given the imperfect Sp of the test, herd size was included as a variable, largely to account for confounding because larger herds would have an inherently greater risk of having false positive test results. In agreement with this, herd size was found to be significant in the logistic model, whereas in the Bayesian model, herd size was not significant. However, previous studies have 
documented increased risk of infection status in association with increased herd size. Collins et al. (1994) found that larger herds in Wisconsin were more likely to be defined positive based on serological methods; however, this association was not statistically significant. Similarly, Daniels et al. (2002) found that clinical disease was more likely to be present on Scottish farms in the preceding $10 \mathrm{yr}$ when herd size was larger. Finally, based on analysis of submitted laboratory samples, Barrett et al. (2011) found a significant association between herd positivity and herd size.

To the authors' knowledge, this study represents the first use of herd level outputs from a Bayesian latent class model to fit a $\beta$ regression on herd-level risk factors. Furthermore, the use of PAF from a classical logistic regression model has not yet been used to investigate the relative importance of risk factors for paratuberculosis. The Bayesian model reduced the risk for misclassification due to imperfect test performance as test Se and Sp were incorporated within the latent class model. On the other hand, the logistic model was based on the binary outcome of assigned herd status facilitated the use of PAF, giving a more intuitive impression of the relative importance of significant risk factors. Both methods are limited by the sampling method in this study. The Irish JD control program is voluntary and therefore may not be representative of the average Irish dairy farm. In addition, the study used a cross-sectional design based on a single test, single RA strategy. Although the recommendation from the national program is to conduct the RA before testing, it is possible that testing may have been conducted before the completion of the RA, prompting the introduction of management changes and thereby introducing the risk of reverse causality into the analysis. The authors attempted to reduce this risk by using RA data from the first year of the program. Given that the management practices identified as significant in this are biologically plausible and largely agree with putative risk factors, it seems unlikely that reverse causality was a significant issue in this analysis.

An unforeseen outcome of the analysis was the strong seasonal effect that was observed in both models. In each model, January, February, and March were associated with a greater risk of positivity. The risk decreased in April, May, and June before increasing again in the autumn and winter. Within the Irish system, seasonality has the potential to be confounded by stage of lactation and therefore milk yield. Nielsen and Toft (2012) found that the risk of testing positive on milk ELISA was greatly increased in the first 7 DIM and increased linearly over the course of the lactation after correcting for milk yield, which appeared to have a diluting effect. To investigate the current data set further, we separated the data set into herds using milk and those using blood. Although the lowest risk month for both data sets was the same (i.e., May), different temporal trends were apparent depending on the test medium used. In the milk data set, the risk steadily increased from March to August with a large peak in September before declining again from September to December. With the serum data set, the highest risk of test positivity was in January with a decline until May, with a second smaller peak in July. These findings require further investigation to examine whether this trend repeats in subsequent years.

\section{CONCLUSIONS}

This study illustrates the use of PAF and Bayesian $\beta$-regression as a means of investigating the relative importance of herd-level interventions on a national scale for the control of paratuberculosis. Results suggest that the national control program should emphasize avoiding the use of the calving pen to house sick or lame cows (or both), reducing the length of time calves spend in the calving pen to less than 15 min and reducing the prevalence of pooled colostrum feeding as key interventions to reduce the prevalence of paratuberculosis in Irish dairy herds. It should also be noted, however, that a large proportion of the observed variation in probability of infection remained unexplained, suggesting other important risk factors may exist.

\section{ACKNOWLEDGMENTS}

This study was carried out as part of the Improved Control of MAP (ICONMAP) multidisciplinary research program funded by the Irish Department of Agriculture, Food and the Marine. The authors acknowledge the assistance of Animal Health Ireland and the Irish Cattle Breeding Federation (Bandon, Co. Cork) in providing data for the study.

\section{REFERENCES}

Barrett, D. J., P. Mee, M. Mullowney, G. Good, T. McGrath, S. Clegg, and S. More. 2011. Risk factors associated with Johne's disease test status in dairy herds in Ireland. Vet. Rec. 168:410.

Branscum, A. J., I. A. Gardner, and W. O. Johnson. 2004. Bayesian modeling of animal-and herd-level prevalences. Prev. Vet. Med. $66: 101-112$.

Branscum, A. J., W. O. Johnson, and M. C. Thurmond. 2007. Bayesian beta regression: Applications to household expenditure data and genetic distance between foot-and-mouth disease viruses. Aus. NZ. J. Stat. 49:287-301.

Cashman, W., J. Buckley, T. Quigley, S. Fanning, S. More, J. Egan, D. Berry, I. Grant, and K. O'Farrell. 2008. Risk factors for the introduction and within-herd transmission of Mycobacterium avium subspecies paratuberculosis (MAP) infection on 59 Irish dairy herds. Ir. Vet. J. 61:464.

Journal of Dairy Science Vol. 100 No. 11, 2017 
Chiodini, R. J., W. M. Chamberlin, J. Sarosiek, and R. W. McCallum. 2012. Crohn's disease and the mycobacterioses: A quarter century later. Causation or simple association? Crit. Rev. Microbiol. 38:52-93.

Christensen, R. W., A. Johnson, T. Branscum, and T. Hanson. 2012. Bayesian Ideas and Data Analysis: An Introduction for Scientists and Statisticians. CRC Press, Boca Raton, FL.

Collins, M. T., W. Sockett, T. Goodger, C. Conrad, D. Thomas, and D. Carr. 1994. Herd prevalence and geographic distribution of, and risk factors for, bovine paratuberculosis in Wisconsin. J. Am. Vet. Med. Assoc. 204:636-641.

Daniels, M., M. Hutchings, D. Allcroft, I. McKendrick, and A. Greig. 2002. Risk factors for Johne's disease in Scotland-The results of a survey of farmers. Vet. Rec. 150:135-139.

Dohoo, I. R., W. Martin, and H. E. Stryhn. 2010. Veterinary Epidemiologic Research. 2nd ed. VER Inc., Prince Edward Island, Canada.

Espejo, L., S. Godden, W. Hartmann, and S. Wells. 2012. Reduction in incidence of Johne's disease associated with implementation of a disease control program in Minnesota demonstration herds. J. Dairy Sci. 95:4141-4152.

Ferrouillet, C., S. Wells, W. Hartmann, S. Godden, and J. Carrier. 2009. Decrease of Johne's disease prevalence and incidence in six Minnesota, USA, dairy cattle herds on a long-term management program. Prev. Vet. Med. 88:128-137.

Gelman, A., Y. Goegebeur, F. Tuerlinckx, and I. Van Mechelen. 2000 Diagnostic checks for discrete data regression models using posterior predictive simulations. J. R. Stat. Soc. C Appl. 49:247-268.

Geraghty, T., D. A. Graham, P. Mullowney, and S. J. More. 2014. A review of bovine Johne's disease control activities in 6 endemically infected countries. Prev. Vet. Med. 116:1-11.

Godden, S., S. Wells, M. Donahue, J. Stabel, J. Oakes, S. Sreevatsan, and J. Fetrow. 2015. Effect of feeding heat-treated colostrum on risk for infection with Mycobacterium avium ssp. paratuberculosis, milk production, and longevity in Holstein dairy cows. J. Dairy Sci. 98:5630-5641

Goff, J. P. 2004. Macromineral disorders of the transition cow. Vet. Clin. North Am. Food Anim. Pract. 20:471-494.

Hanley, J. A. 2001. A heuristic approach to the formulas for population attributable fraction. J. Epidemiol. Community Health 55:508-514.

Hendrick, S. H., D. F. Kelton, K. E. Leslie, K. D. Lissemore, M. Archambault, and T. F. Duffield. 2005. Effect of paratuberculosis on culling, milk production, and milk quality in dairy herds. J. Am. Vet. Med. Assoc. 227:1302-1308.

Jago, J. G., and D. P. Berry. 2011. Associations between herd size, rate of expansion and production, breeding policy and reproduction in spring-calving dairy herds. Animal 5:1626-1633.

Johnson-Ifearulundu, Y., J. Kaneene, D. Sprecher, J. Gardiner, and J. Lloyd. 2000. The effect of subclinical Mycobacterium paratuberculosis infection on days open in Michigan, USA, dairy cows. Prev. Vet. Med. 46:171-181.

Khol, J. L., V. Beran, P. Kralik, M. Trckova, and I. Pavlik., I. and W. Baumgartner. 2010. Grass silage contaminated with Mycobacterium avium subspecies paratuberculosis (MAP): A possible source of paratuberculosis infection in ruminants? Vet. Med. Czech $55: 225-232$.

Kudahl, A. B., S. S. Nielsen, and S. Østergaard. 2011. Strategies for time of culling in control of paratuberculosis in dairy herds. J. Dairy Sci. 94:3824-3834.

Lu, Z., Y. H. Schukken, R. L. Smith, and Y. T. Grohn. 2010. Stochastic simulations of a multi-group compartmental model for Johne's disease on US dairy herds with test-based culling intervention. J. Theor. Biol. 264:1190-1201.

Lunn, D. J., A. Thomas, N. Best, and D. Spiegelhalter. 2000. WinBUGS - A Bayesian modelling framework: Concepts, structure, and extensibility. Stat. Comput. 10:325-337.

McAloon, C. G., M. L. Doherty, P. Whyte, L. O'Grady, S. J. More, L. L. M. Messam, M. Good, P. Mullowney, S. Strain, and M. J. Green. 2016a. Bayesian estimation of prevalence of paratuberculosis in dairy herds enrolled in a voluntary Johne's Disease Control Programme in Ireland. Prev. Vet. Med. 128:95-100.
McAloon, C. G., P. Whyte, S. J. More, M. J. Green, L. O'Grady, A Garcia, and M. L. Doherty. 2016b. The effect of paratuberculosis on milk yield-A systematic review and meta-analysis. J. Dairy Sci. 99:1449-1460.

McAloon, C. G., P. Whyte, S. J. More, L. O'Grady, and M. L. Doherty. 2015. Development of a HACCP-based approach to control paratuberculosis in infected Irish dairy herds. Prev. Vet. Med. 120:152-161.

McGuirk, S. M., and M. Collins. 2004. Managing the production, storage, and delivery of colostrum. Vet. Clin. North Am. Food Anim. Pract. 20:593-603.

Messam, L. L. M., A. J. Branscum, M. T. Collins, and I. A. Gardner 2008. Frequentist and Bayesian approaches to prevalence estimation using examples from Johne's disease. Anim. Health Res. Rev. 9:1-23.

More, S. J., M. L. Doherty, L. Downey, K. McKenzie, C. Devitt, and J. O'Flaherty. 2011. Animal Health Ireland: Providing national leadership and coordination of non-regulatory animal health issues in Ireland. Rev. Sci. Tech. 30:715.

Mortier, R. A., H. W. Barkema, J. M. Bystrom, O. Illanes, K. Orsel, R. Wolf, G. Atkins, and J. De Buck. 2013. Evaluation of agedependent susceptibility in calves infected with two doses of $M y c o-$ bacterium avium subspecies paratuberculosis using pathology and tissue culture. Vet. Res. (Faisalabad) 44:94.

Nielsen, S. S., H. Bjerre, and N. Toft. 2008. Colostrum and milk as risk factors for infection with Mycobacterium avium subspecies paratuberculosis in dairy cattle. J. Dairy Sci. 91:4610-4615.

Nielsen, S. S., and N. Toft. 2012. Effect of days in milk and milk yield on testing positive in milk antibody ELISA to Mycobacterium avium subsp. paratuberculosis in dairy cattle. Vet. Immunol. Immunopathol. 149:6-10.

Nielsen, S. S., N. Toft, and H. Okura. 2013. Dynamics of specific antiMycobacterium avium subsp. paratuberculosis antibody response through age. PLoS One 8:63009.

Obasanjo, I. O., Y. Gröhn, and H. O. Mohammed. 1997. Farm factors associated with the presence of Mycobacterium paratuberculosis infection in dairy herds on the New York State Paratuberculosis Control Program. Prev. Vet. Med. 32:243-251.

Pillars, R. B., D. Grooms, J. Gardiner, and J. Kaneene. 2011. Association between risk-assessment scores and individual-cow Johne's disease-test status over time on seven Michigan, USA dairy herds. Prev. Vet. Med. 98:10-18.

Pithua, P., S. M. Godden, S. J. Wells, and M. J. Oakes. 2009. Efficacy of feeding plasma-derived commercial colostrum replacer for the prevention of transmission of Mycobacterium avium subsp paratuberculosis in Holstein calves. J. Am. Vet. Med. Assoc. 234:11671176 .

Pithua, P., S. M. Godden, S. J. Wells, and J. R. Stabel. 2011. Evaluation of the risk of paratuberculosis in adult cows fed Mycobacterium avium subsp paratuberculosis DNA-positive or-negative colostrum as calves. Am. J. Vet. Res. 72:1456-1464.

R Core Team. 2016. R: A language and environment for statistical computing. R Foundation for Statistical Computing, Vienna, Austria. https://www.R-project.org/.

Richardson, E., and S. J. More. 2009. Direct and indirect effects of Johne's disease on farm and animal productivity in an Irish dairy herd. Ir. Vet. J. 62:526.

Robins, J., A. Bogen, A. Francis, A. Westhoek, S. Kanarek, S. Lenhart, and S. Eda. 2015. Agent-based model for Johne's disease dynamics in a dairy herd. Vet. Res. 46:68.

Soder, K. J., and W. L. Stout. 2003. Effect of soil type and fertilization level on mineral concentration of pasture: Potential relationships to ruminant performance and health. J. Anim. Sci. 81:1603-1610.

Sorge, U. S., K. Lissemore, A. Godkin, J. Jansen, S. Hendrick, S. Wells, and D. Kelton. 2011. Changes in management practices and apparent prevalence on Canadian dairy farms participating in a voluntary risk assessment-based Johne's disease control program. J. Dairy Sci. 94:5227-5237.

Stabel, J. R. 2008. Pasteurization of colostrum reduces the incidence of paratuberculosis in neonatal dairy calves. J. Dairy Sci. 91:36003606 
Sweeney, R. W., M. T. Collins, A. P. Koets, S. M. McGuirk, and A. J. Roussel. 2012. Paratuberculosis (Johne's disease) in cattle and other susceptible species. J. Vet. Intern. Med. 26:1239-1250.

Trotz-Williams, L. A., S. W. Martin, K. E. Leslie, T. Duffield, D. V. Nydam, and A. S. Peregrine. 2008. Association between management practices and within-herd prevalence of Cryptosporidium parvum shedding on dairy farms in southern Ontario. Prev. Vet. Med. 83:11-23.

Weary, D. M., and B. Chua. 2000. Effects of early separation on the dairy cow and calf: 1 . Separation at $6 \mathrm{~h}, 1$ day and 4 days after birth. Appl. Anim. Behav. Sci. 69:177-188.

Wells, S. J., and B. A. Wagner. 2000. Herd-level risk factors for infection with Mycobacterium paratuberculosis in US dairies and asso- ciation between familiarity of the herd manager with the disease or prior diagnosis of the disease in that herd and use of preventive measures. J. Vet. Med. Assoc. 216:1450-1457.

Whittington, R. J., D. J. Marshall, P. J. Nicholls, I. B. Marsh, and L. A. Reddacliff. 2004. Survival and dormancy of Mycobacterium avium subsp. paratuberculosis in the environment. Appl. Environ. Microbiol. 70:2989-3004.

Windsor, P. A., and R. J. Whittington. 2010. Evidence for age susceptibility of cattle to Johne's disease. Vet. J. 184:37-44.

Zhang, J., and F. Y. Kai. 1998. What's the relative risk?: A method of correcting the odds ratio in cohort studies of common outcomes. JAMA 280:1690-1691. 